\title{
Research on the Diffusion of Network Buzzwords from the Perspective of Communication
}

\author{
Ruiyang Qu*
}

Zhejiang University of Finance \& Economics Dongfang College, Jiaxing 314400, China. E-mail: 1026927958@qq.com

Abstract: With the advent of the era of big data, the popularity of "Internet + " has promoted the development of the social and cultural environment, and the production and dissemination of information have also had an important impact on social culture. As the communication language of Internet users in the network culture, the emergence and development of network catchwords also reflects the psychological demands of the audience and the direction of cultural communication in the digital era under the influence of the development of the Internet. From this perspective, from the perspective of communication, this paper discusses the new development trend of the way of the spread of Internet buzzwords in the context of the development of the times, and its impact.

Keywords: Internet Buzzwords; Diffusion; Communication

\section{Brief description}

\subsection{Internet buzzwords}

In a sense, the act of using a certain language is an attribution and recognition of the group to which it belongs, and the discourse used is also a marker of group attributes ${ }^{[1]}$. Internet buzzwords, as the communication language of the majority of netizens in the Internet culture, to a certain extent reflect the psychological demands of the audience and the direction of cultural propagation in the digital age. Internet buzzwords are different from online languages, but also cross new words. It is not easy to define it clearly. There is currently no unified concept for Internet buzzwords in the academic world, and the concept of Internet buzzwords that we are going to talk about here can be defined as: the most active, fermenting, and special use that is spontaneously used by netizens in the Internet field within a certain period of time meaningful language signs that often have an impact on social reality. ${ }^{[2]}$

\subsection{Evolution}

Internet buzzwords have developed along with the development of the Internet, from being initially unacceptable to the public, and now being integrated into people's lives and closely related. The development of Internet technology has brought people new ways of communication and communication platforms. Because of their humorous, concise, practical, and profound meanings, Internet buzzwords have a strong communication power on social networks, gradually changing from the non-mainstream forms of the past. It is now recognized and accepted by mainstream media and the public. As a popular language expression, Internet buzzwords, regardless of their language content, expression and communication mechanism, reflect or reflect the social circumstances and social mentality of netizens.

With the popularization of emerging information publishing platforms such as WeChat, Weibo, QQ, etc., Internet buzzwords are more closely connected with the daily life of the public, and naturally become the public's personal comments on the current popular social culture and phenomena. View tool. Therefore, today's Internet buzzwords not only remain at the early level of singularity or satisfying identity, but also shoulder more of the netizens' tools of

This is an open-access article distributed under the terms of the Creative Commons Attribution Non-Commercial License (http://creativecommons. org/licenses/by-nc/4.0/), which permits unrestricted non-commercial use, distribution, and reproduction in any medium, provided the original work is properly cited. 
criticism, supervision and other functions of social issues. The main sources of online buzzwords are social hotspots, content and events, foreign cultures, popular film and television variety shows, and old new words.

\section{Factors and effects}

Look for the rules of the development of Internet buzzwords, analyze the spread of Internet buzzwords and their impact, and explore ways to effectively control web buzzwords in the digital age. No matter what kind of social sentiment the public feels about internet popularity, and the internet buzzwords satisfy the public's He Zhong emotion, it is undeniable that it has already had a certain impact on people's lives.

\subsection{Factors in the spread of Internet buzzwords}

The vitality of language lies in the needs of communication. Only those universal network catchwords which are inseparable from people's production and life and can fully express people's feelings can have strong vitality and be inherited for a long time, and finally become a part of the vast ocean of national language and culture. ${ }^{[3]}$

\subsubsection{Media factors}

Information dissemination such as Internet buzzwords is the dissemination of information through various social media such as BBS, Tieba, forum, QQ, WeChat and so on. In the era of Web2.0, with the widespread application of social media such as Weibo and WeChat, UGC (user-generated content) was quickly accepted by netizens with its personalization, grassroots and fun, forming a unique discourse system in the Internet environment (Professional production content) join forces to form a colorful cultural landscape. ${ }^{[4]}$ Nowadays, "media integration" is the general trend and an irreversible general trend. The integration of traditional media and new media and complementary advantages provide an important platform for the public to understand all kinds of information, express personal attitudes and participate in social affairs. The more complete communication channel formed by "media fusion" has also promoted the development and spread of online buzzwords.

\subsubsection{Use group factors}

According to the 45th "Statistical Report on Internet Development in China" released by CNNIC, as of June 2019, the number of Internet users in China was 904 million, the number of mobile Internet users reached 897 million, and the proportion of Internet users using mobile phones to access the Internet reached $99.3 \% .{ }^{[5]}$ Almost $100 \%$ of the Internet access on mobile phones shows that the scale of Internet users is huge and the power of public opinion will be exerted to the extreme. It can be seen from Figure 1 and Figure 2 that netizens are mainly young and middle-aged, and the netizens have a medium level of education, generally showing the characteristics of low education and low age. And this part of the population is susceptible to the influence of the environment, and driven by public opinion, it is likely to become a member of the "a motley crew".

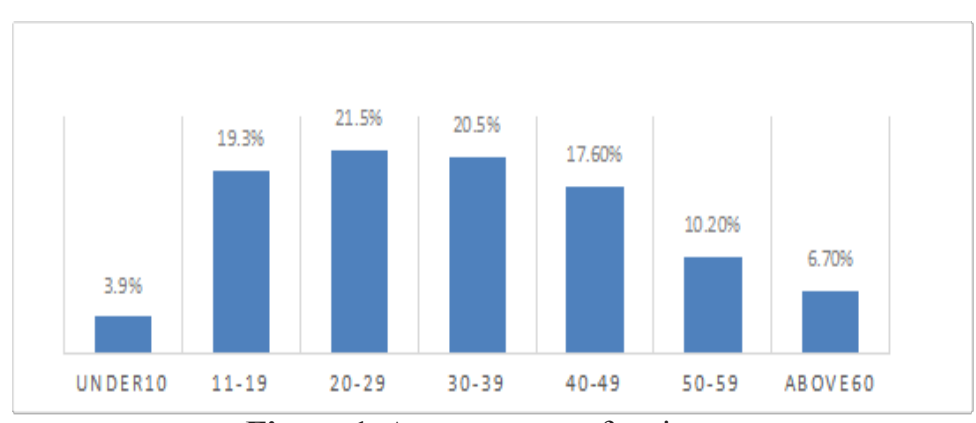

Figure 1. Age structure of netizens. 


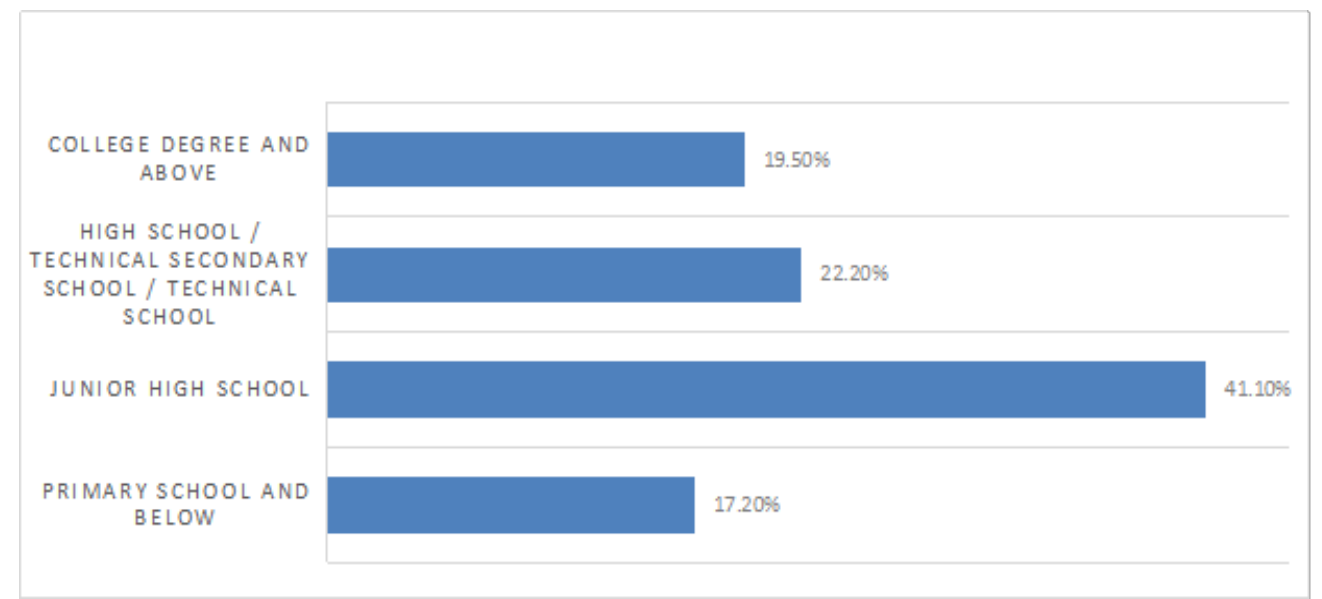

Figure 2. Education structure of netizens.

\subsubsection{Content factors}

Innovation is a basic element of Internet buzzwords. Any type of it has a new idea that is different from traditional languages, but most of them are based on traditional languages. With the development of society and the advancement of science and technology, new vocabulary continues appeared, this innovation was first reflected on the Internet, and then extended from the Internet to the public life, and was eventually accepted by the academic community. Because the Internet buzzwords are mostly used by people with low education and low age, they have great creativity and curiosity about new things, and the content of the dissemination is simple and convenient, which makes people at a glance, such as "PK", "886", "Happy"content, high usage rate, easy to understand. There is also a kind of content such as "porridgelike", "group pig-group owner", homophonic words derived from original words such as words, phrases or English words, and the network words after mutation, the meaning and expression of the literal meaning is quite different, but its popularity and usage environment determine that most netizens will not be ambiguous.

\subsection{Effect and influence}

As a popular language for all people, Internet buzzwords have the characteristics of concise language, closeness to life, high openness, and strong interactivity, but also have the characteristics of subculture, blind obedience and high imitation, that is, the spread of Internet buzzwords. It covers all aspects of social life in the digital age. ${ }^{[6]}$ I divide the influence of Internet buzzwords into positive influence and negative influence.

From the perspective of positive influence, the network catchwords are very interesting and can make the network cultural environment more colorful, and netizens can also vent their bad emotions through self ridicule, banter, teasing and other ways, such as "I'm too difficult", "what kind of rubbish are you", "farmer's wife, mountain spring, a little field" and other network catchwords, which can help relieve pressure and release emotions in a humorous way. And in certain circumstances, it has certain economic value. Just like "can’t afford to hurt", "god horse is floating cloud", these songs are featured by advertising sponsorship, fee download and other ways to bring economic benefits. It is also conducive to public opinion monitoring by relevant departments.

From the perspective of negative influence, Internet buzzwords have the characteristics of fast spread and large influence. It has the characteristics of bursting in a short time, and its emotional color is easily changed. In the process of spreading the Internet popular body, because of its entertainment and spoofing characteristics, it is easy to cause the joke of the Internet spree ${ }^{[7]}$ For example, self-regulation is used to release pressure during positive influence, but if the method or content of publication is not appropriate, it will cause a bad influence. The Internet has a certain degree of anonymity. With the spread among netizens, it will violate the rights of the subjects involved in the initiation of Internet buzzwords and cause unavoidable harm. Due to its changeable emotional color, some vulgar violence content may also be understood by the public through this channel, affecting the social atmosphere and bringing about undesirable consequences.

\section{Control}


The public should take the attitude of "taking the best of its essence and removing its dross" for the spread of Internet buzzwords. Because Internet buzzwords have the characteristics of fast spread and high influence, if they affect the social order due to their spread, they should be it performs proper control. Therefore, I mainly from three aspects of audience control, political control, media control. To analyze the control of the spread of Internet buzzwords.

\subsection{Audience control}

Qingguang Guo pointed out in "Communication Course": "In modern society, mass communication has an important impact on all aspects of people's social life. Many communication contents, such as whether the reported news or the information disseminated are true, whether the advertisements published reliability, whether the provided cultural and entertainment content is healthy and beneficial, etc., will directly affect the interests of the audience. Therefore, the general audience has a legitimate right to conduct social supervision of the activities of the media." ${ }^{[8]}$ Therefore, the audience plays an important role in the control of Internet buzzwords. Therefore, the audience should avoid the herd psychology, maintain rational thinking on the network, and effectively control the audience's reception and delivery of messages.

\subsection{Policy control}

In the face of the development of Internet buzzwords, most scholars and experts maintain a rational attitude towards the development of Internet buzzwords, believing that Internet buzzwords require the introduction of relevant policies to regulate their good development. Therefore, I believe that policy control is an indispensable means. National agencies should formulate relevant legal policies through legislative work, strengthen network monitoring, limit the scope of Internet buzzwords, and establish standards for Internet buzzwords that can enter real life. It is strictly forbidden to use vulgar, violent or internet buzzwords with bad thoughts and tendencies, and to prohibit and punish all behaviors that adversely affect others and society through the hype of internet buzzwords.

\subsection{Media control}

Internet buzzwords are a special language, which is an extension of modern languages. The emergence of Internet buzzwords is inseparable from the Internet. The Internet is the birthplace of Internet buzzwords. ${ }^{[9]}$ Moreover, when the public opinion is absent from the system, it has assumed the role of supervising power. The media is an important way to spread and develop online buzzwords. Therefore, from the media level, it is necessary to publicize the importance of using online buzzwords through various forms of media at all levels, while strengthening publicity. The concept of standardizing the use of Internet buzzwords can penetrate people's hearts.

\section{Conclusion}

In this era of Internet, everything is changing, and the development of network catchwords is increasingly diversified, its characteristics and types are also rich, with a good space for communication and development. But everything has two sides. The courage of its development brings unavoidable negative effects. We should look at this problem from different angles. The public should maintain the attitude of "taking the essence and eliminating the dross" and rationally treating it. And if it is controlled and used effectively, the social development will be further.

\section{References}

1. Jiang X, Li M, An J. Space switching: The social mechanism for the production of online buzzwords (in Chinese). The Journal of Humanities 2015; (12): 119-125. doi: 10.15895/j.cnki.rwzz.2015.12.017.

2. Wang S. Discrimination and analysis of the concepts and features of Internet buzzwords (in Chinese). Probe 2014; (4): 186-192. doi: 10.3969/j.issn.1007-5194.2014.04.035.

3. Wang S. On the development of Internet buzzwords and countermeasures (in Chinese). Way of Success 2020; (10).

4. Wang G. Diffusion mechanism of Internet buzzwords from the perspective of communication (in Chinese). 2019; (12).

5. Data collected from CNNIC, and the due date is 2020-04-28.

6. Luo C, He T. An analysis of the evolution of Chinese Internet catchword and its effect on digital cultural 
communication. Journal of Henan University (Social Science) 2015; 55(2): 108-115.

7. Cui H. Research on the causes, mechanisms and effects of Internet popularity dissemination (in Chinese).

8. Guo Q. Communication course.

9. Yan W. Research on the application of Internet buzzwords in People's Daily (2012-2016). Henan University 2018. 